\title{
Diversity of occupants' activity impact on indoor air
}

\author{
Andrzej Szczurek ${ }^{1}$, Monika Maciejewska ${ }^{1, *}$, and Anna Dolega ${ }^{1}$ \\ ${ }^{1}$ Wrocław University of Science and Technology, Faculty of Environmental Engineering, \\ Wybrzeże Wyspiańskiego 27, 50-370 Wrocław, Poland
}

\begin{abstract}
The problem of indoor air quality (IAQ) has attracted a great deal of attention recently. One of interesting aspects of this problem is the characterization of human impact on IAQ. This work presents a method which allows to determine the diversity of building occupants influence on indoor air. The method is based on the supervised clustering of the indoor air measurement data in domains of various indoor air parameters. The introduced approach was applied to examine the exemplary data set. The diversity of impact was studied in the domain of air parameters which characterize thermal conditions indoors and in the domain of chemical parameters of indoor air. The obtained results showed that the diversity of impact was greatly related to the fact that occupants' activities occurred in combinations, rather than individually. It was also demonstrated that the diversity of impact was domain dependent. Most of examined activities showed greater diversity of influence in the domain of parameters which characterize thermal conditions. This shows that the activity-specific impacts would be rather associated with the domain of chemical parameters of indoor air. The proposed method allows to obtain information which can be useful in the diagnostics of IAQ and for the control of ventilation.
\end{abstract}

\section{Introduction}

Over the last 30 years, an increasing number of complaints about discomfort and health effects related to indoor air quality (IAQ) have been reported [1-3]. The term IAQ refers to chemical, biological and physical contamination of air in indoor spaces. Negative opinions arrive from different environments, e.g. residential, occupational, and institutional settings. Sometimes, indoor air quality makes up a greater hazard than outdoor air pollution [4]. In particular, it affects health, safety, productivity and comfort of occupants [5]. Hence, the issue of IAQ has attracted a great deal of attention recently [6]. The improvement of the current situation requires that a broad range of issues are discussed, for example the problem of recognition of factors influencing air inside buildings.

Human environment is a collection of components that interact with each other to form some aggregated whole. The close coupling and interactions between the components of this complex system cause recognizable collective behavior [7]. Hence, IAQ may be seen

\footnotetext{
* Corresponding author: monika.maciejewska@pwr.edu.pl
} 
as the product of numerous internal and external factors as well as decentralized and local interactions. It is affected by meteorological conditions, the interaction between the building and its surrounding, infiltration, indoor and outdoor pollutants sources, building characteristics, operation and maintenance of the heat, ventilation and air conditioning (HVAC) system, as well as occupancy [8]. Air quality indoors can change sharply due to occurrences such as the presence of people, the use of air conditioners and radiators, weathering etc. [9]. The information about these influences has a fundamental significance for building managers, policy makers, health professionals and researchers. It is crucial for building commissioning, proactive building management, diagnostics of indoor air quality complaints, and investigation of building energy consumption.

This work was focused on human impact on IAQ. This influence is an effect of occupants themselves (their presence) and their activities in terms of kind (e.g. tobacco smoking, use of cleaning products, plant and pet drugs, cooking etc.) as well as the temporal and spatial characteristics which may be recognized as the living pattern or lifestyle [10]. Human impact on IAQ can be analysed from different perspectives. The most popular one is the detection of occupants activities based on the 'footprint' they leave in environmental [11-13]. In this paper, we present the method which allows to determine the diversity of occupancy influence on IAQ. In our opinion, the information obtained in this way can be useful in the diagnostics of IAQ and for the control of ventilation.

\section{Experimental part}

The study was based on two sources of data: the measurements of selected parameters of indoor air and the observation of building occupants' activities.

Sensor devices were applied for measurement data collection. The first group of sensors was dedicated to the measurement of temperature $(\mathrm{T})$ and relative humidity $(\mathrm{RH})$. These sensors allow to characterize thermal conditions indoors. Temperature was measured with thermoresistor NTC $10 \mathrm{k}$ characterised by: measuring range $-20 \ldots 60^{\circ} \mathrm{C}$, accuracy $\pm 0.2^{\circ} \mathrm{C} \pm 0.15 \%$, resolution $0.1^{\circ} \mathrm{C}$. Relative humidity was measured with the capacitive sensor characterised by: measuring range $5 \ldots 100 \%$, accuracy $\pm 2 \%$ in the range $10-90 \%$ $\mathrm{RH}$, resolution $0.1 \% \mathrm{RH}$.

The second group of sensors comprised semiconductor gas sensors. These partially selective devices provide chemical information. It refers to the qualitative and quantitative composition of VOCs mixtures in air. The sensors are not dedicated to the selective measurement of any particular component of air. The following sensors were used: TGS8100, TGS2600, TGS2602, TGS2603, TGS2610, TGS2611 and TGS2620. These commercially available devices are produced by Figaro Eng. [24].

The experimental study took place in the flat occupied by a young family (parents and a child). The flat consists of two rooms, kitchen, corridor and bathroom. It was assumed, that the impact of occupants on indoor air is reflected in the measurement data collected in the flat.

The monitoring was accomplished in a small room, in the period from 5.12.2016 to 08.01.2017 (during 14 days). Continuous measurements ran for several hours a day. The data analysed in this work was collected in the total time of 63 hours. The data recording resolution was $1 \mathrm{~s}$.

The following activities were observed in the flat during the study: 1 . weathering by keeping small room window opened, 2. weathering by keeping living room window opened, 3 . weathering by keeping kitchen window opened, 4 . weathering by keeping small room door opened, 5. heating by using oil filled electric radiator, 6 . heating by using electric heater with fun blower, 7. heating by using convector heater, 8. wet dusting, 
9. vacuum cleaning, 10. wet mopping, 11. washing, 12. doing bed, 13. wall painting, 14. playing with the child, 15. changing diaper, 16. getting child changed, 17. dressing up for a walk, 18. child bath, 19. water boiling, 20. cooking, 21. frying, 22. eating, 23. air-freshening with electrical device, 24. keeping flat decorated with the Christmas tree, 25. use of cosmetics, 26 smoker visit in the flat.

\section{Methods}

Various activities of occupants have an impact on indoor air. Based on observations, people rarely undertake one activity a time when staying indoors. Most often, several activities occur in parallel. For example, the flat is weathered and cleaned at the same time, while the washing is on. As a result, a group of activities influences indoor air more often than a single activity. Due to numerous possible activities of occupants, the number of their potentially occurring combinations is huge. Most of people tend to think about the impact of a particular activity rather than about the impact of it in combinations with other activities. This kind of simplification may not be justified.

In this work we proposed a method to analyse the diversity of impact of occupants activities, which results from their co-occurrence with other activities. The method is based on the measurements of the selected quantities characterizing indoor air. The measurements shall be performed when various activities take place indoors.

The principal element of our concept was centroid. The centroid of the $K$ dimensional data set, which includes $L$ data points is defined as the vector $\boldsymbol{x}=\left[x_{k}\right]$ where

$$
x_{k}=(1 / L)\left(x_{k 1}+x_{k 2}+\ldots+x_{k L}\right)
$$

where $k=1 . . K$ and $l=1 \ldots L$. The centroid is the central point for the data set. It is considered as the most representative for the group of data points.

In this work, we dealt with the measurement data which consisted of all measurements performed in the period of study. The set was defined in the multidimensional space of the parameters of indoor air. Individual data points in this set were results of single measurements, done with the temporal resolution of $1 \mathrm{~s}$. The data set was divided into subsets. A subset included all measurement data which was recorded when the particular combination of occupants activities occurred in indoor space. For such data subset the centroid was determined. The centroid indicated the impact of a particular combination of occupants activities on indoor air.

A particular activity could occur in multiple combinations with others. Each of such combinations had the associated centroid. Each centroid reflected the influence of the activity on indoor air. Centroids together formed a cluster. It was proposed to consider the size of the cluster as the indication of the diversity of impact of a particular activity on indoor air. The cluster size was represented by the maximum Euclidean distance between centroids in the cluster.

If $\boldsymbol{x}_{i}=\left(x_{i, 1}, x_{i, 2}, \ldots, x_{i, k}\right)$ and $\boldsymbol{x}_{\boldsymbol{j}}=\left(x_{j, 1}, x_{j, 2}, \ldots, x_{j, k}\right)$ are two centroids in the $K$ dimensional Euclidean space, the Euclidean distance between two centroids is:

$$
D\left(x_{i}, x_{j}\right)=\left(\left(x_{i, 1}-x_{j, 1}\right)^{2}+\left(x_{i, 2}-x_{j, 2}\right)^{2}+\ldots+\left(x_{i, k}-x_{j, k}\right)^{2}\right)^{1 / 2}
$$

The impact diversity indicator was defined as the ratio of the Euclidean distance between the most distant centroids in the cluster and the maximum between-centroids distance in the full measurement data set. This solution allows to compare the diversity of impact in different domains, namely the domains of different indoor air parameters. 


\section{Results}

The presented method was applied to examine the diversity of household activities impact on indoor air. The results of analysis were presented in Table 1 and in Fig. 1 to Fig. 4. Table 1 displays all combinations of occupants activities which were observed indoors and their overall time of occurrence in the measurement period. Fig. 1 shows the sizes of clusters of centroids for individual activities of occupants. Clusters' dimensions were determined in the domain of parameters which represent thermal conditions and in the domain of parameters which characterize the chemical quality of indoor air. Fig. 2 presents the relationship between the size of clusters and the number of centroids in the clusters. Fig. 3 focuses on individual activities and it compares the size of the associated clusters in two domains of indoor air parameters. Finally, Fig. 4 examines the relationship between the sizes of clusters in the two domains.

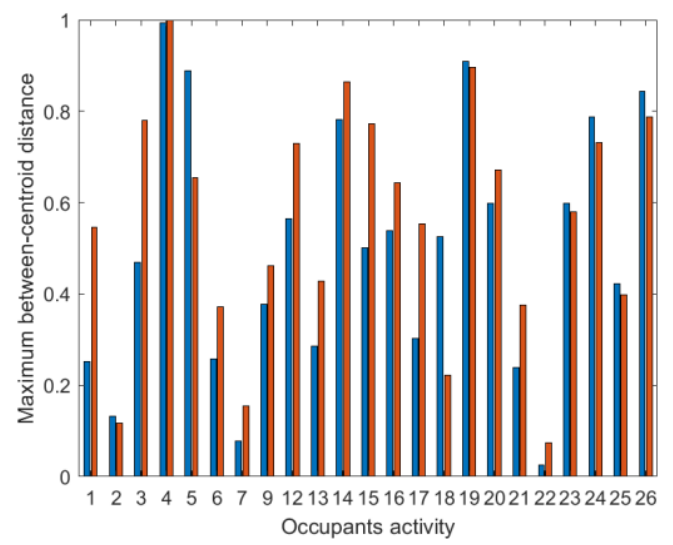

Fig. 1. The size of centroids clusters associated with individual activities of occupants. Domain of parameters representing thermal conditions (red); Domain of parameters which characterize the chemical quality of indoor air (blue). The utilised IDs of activities were explained in Sec. 2.
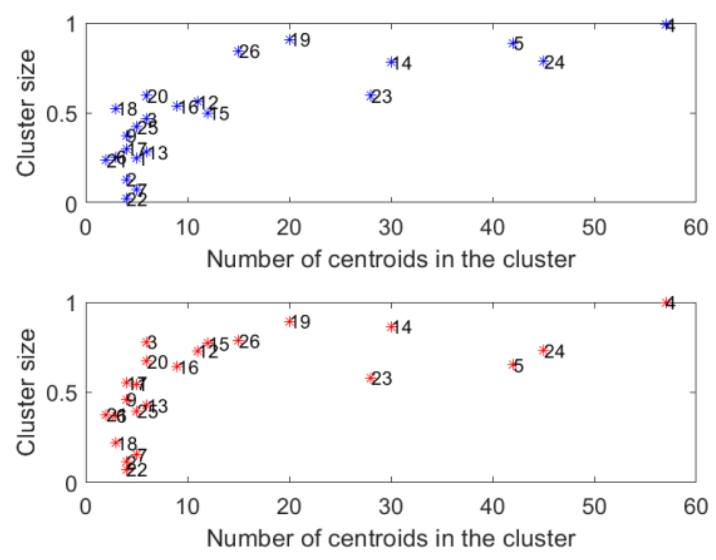

Fig. 2. Cluster size as a function of the number of centroids in the cluster. The utilised IDs of activities were explained in Sec. 2. Domain of parameters which characterize the chemical quality of indoor air (top); Domain of parameters which describe thermal conditions (bottom). 
Table 1. Occupants' activities, labelled from 1 to 23 and their combinations, labelled from $\mathrm{C} 1$ to C105. For each combination, the time of occurrence was quoted.

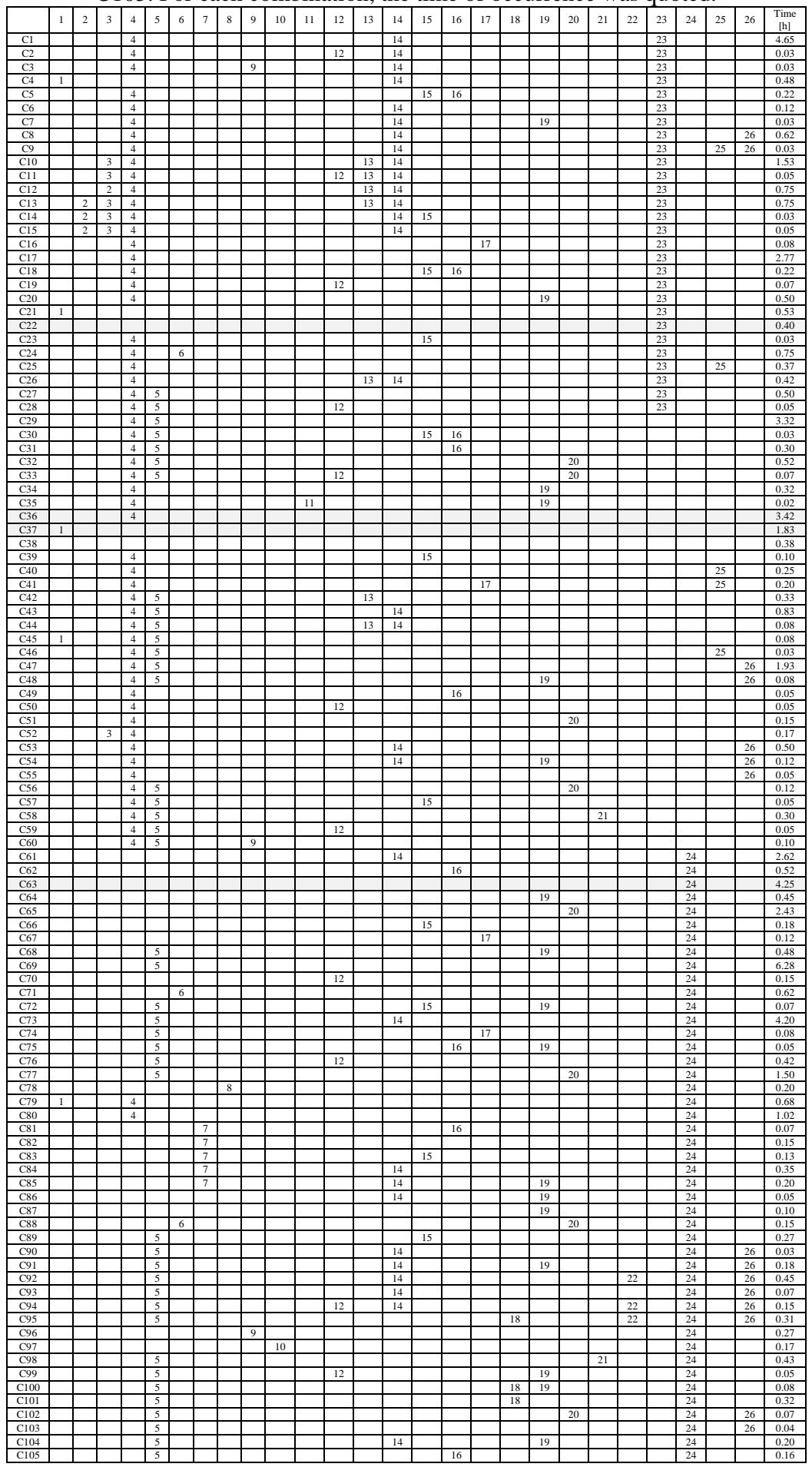




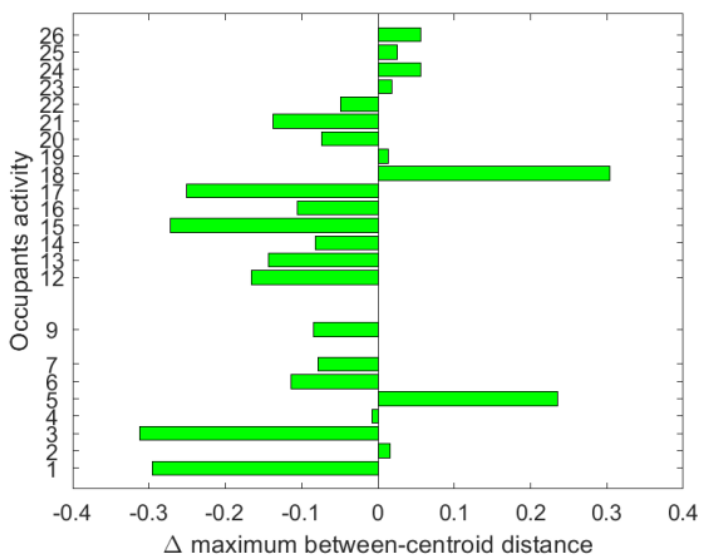

Fig. 3. The difference between the size of the cluster in the domain of parameters related to chemical quality of indoor air and in the domain of parameters related to thermal conditions. Differences were computed for all activities. The utilised IDs of activities were explained in Sec. 2.

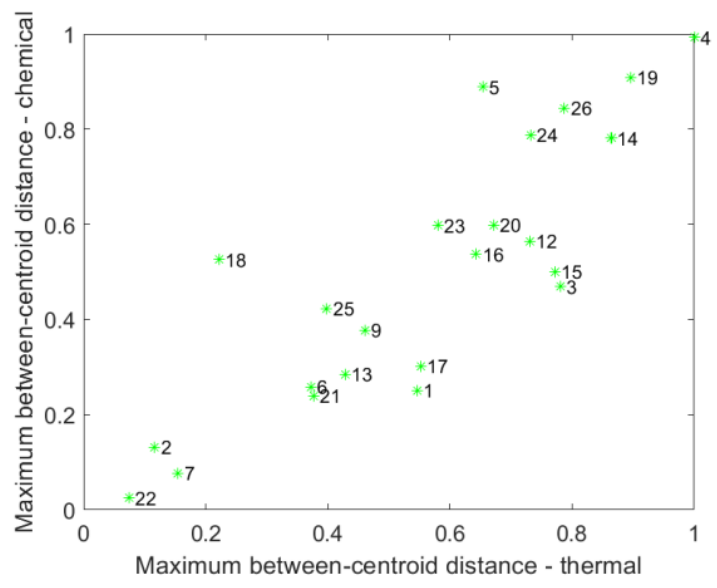

Fig. 4. The relationship between the sizes of clusters in two domains, the domain of parameters representing thermal conditions and the domain of parameters related to chemical quality of indoor air. The utilised IDs of activities were explained in Sec. 2.

As shown in Table 1, 26 occupants activities were observed in the measurement period. The number of theoretically possible combinations of these activities is $2^{26}=67108864$. In the measurement period there were identified 105 unique combinations of occupants activities. The observed combinations constituted a very small fraction of the total, namely $0.0016 \%$. They were composed of $0,1,2,3,4,5$ or 6 activities. The most plentiful were three-component combinations (41). One-component combinations were very rare (4).

As shown in Fig. 1, the clusters of centroids had various size depending on the associated activity. This indicates that individual activities had different diversities of impact. The most extensive clusters were observed for activities: 4. weathering by keeping small room door opened, 5. heating by using oil filled electric radiator, 14. playing with the child, 19. water boiling, 23. air-freshening with electrical device, 24. keeping flat decorated with the Christmas tree, 26 smoker visit in the flat. These activities exhibited largest diversity of impact on indoor air. The least extensive clusters were observed for activities: 
2. weathering by keeping living room window opened, 7 . heating by using convector heater, 22. Eating. These activities had smallest diversities of impact.

To some extent, the diversity of impact may be attributed to the number of combinations in which the activity participated. This aspect was examined in Fig. 2. As shown, the size of the cluster was related to the number of centroids in the cluster. Namely, two groups of activities could be distinguished. The first group consisted of activities which occurred in less than 15 different combinations and the other group included activities which were elements of more than 15 combinations. The maximum between-centroid distance in the first group was less than 0.6. In the second group the distance was grater. Based on these results, one would not expect that the activity which occurs in large number of combinations has small diversity of impact. The opposite case is more likely. However, the number of centroids did not determine the size of cluster. As shown in Fig. 2, activities, which participated in a similar number of combinations had clusters of various sizes, i.e. various diversities of impact. We show further that the diversity of impact was also dependent on the domain of analysis.

Fig. 3 compares the diversities of impact for individual activities in two domains of indoor air parameters. It was shown, that the maximum between-centroid distances were different in the domain of parameters describing chemical quality of indoor air and in the domain of parameters which refer to thermal comfort. Majority of activities (15) displayed greater diversity of impact in the domain related to thermal conditions. In case of 8 activities, the impact was more diverse in the domain of parameters of chemical quality of indoor air. Activities, for which the diversity of impact was greater in the domain of microclimate parameters had more consistent impact on chemical quality of indoor air. In this domain, their influence on indoor air was less susceptible to the co-occurring activities. The fact that domain-related differences in the diversity of impact exist is important. The domain with zero diversity of impact for a particular activity would be the domain of the unique, indicative impact of this activity. As shown by the obtained results, for most of occupants activities, their indicative impact would be rather occurring in the domain of chemical parameters of indoor air. However, the domain accounting for chemical parameters, chosen for consideration in this work was not optimal in this respect. As shown in Fig. 4, there was a considerable covariance of the cluster size in this domain and in in the domain of parameters related to thermal conditions indoors.

\section{Conclusions}

The work presents a method to analyse the diversity of impact of occupant's activity on indoor air. The diversity results from joint occurrence of multiple activities. The method was based on the measurements of indoor air parameters and observation of the occurrence of human activities.

The key element of the method was the cluster of centroids. Centroid represented the subsets of the measurement data, which were collected when the defined combination of occupants activities was observed. Cluster included all centroids associated with a particular activity. The size of cluster was indicative for the diversity of impact of the activity.

Based on the presented results, the occurrence of occupants' activities in combinations, rather than individually, resulted in the nonzero diversity of impact. The diversity was dependent on the domain of determination. For most activities the diversity of impact was larger in the domain of air parameters which characterize thermal conditions indoors. Hence, the activity-specific impacts would be rather associated with the domain of chemical parameters of indoor air. 
The presented approach may be extended to other features of the measurement data, different from single measurements. These would offer new domains where the unique impact of individual activities could be identified.

The real challenge is to examine the diversity of impact in respect of the composition of clusters of mutually occurring activities. This is one of our future goals.

\section{References}

1. S. Burge, A. Hedge, S. Wilson, J. H. Bass, A. Robertson, Ann. Occup. Hyg. 31, 4A (1987)

2. N. Zhang, Y. Kang, K. Zhong, J. Liu, Indoor Built Environ. 25, 4 (2016)

3. H. R. Bohanon, J. J. Piadé, M. K. Schorp, Y. Saint-Jalm, J. Expo. Anal. Env. Epid. 13, (2003)

4. World Health Organization WHO. Guidelines for air quality. World regional publication European series No. 91, $2^{\text {nd }}$ ed. Geneva (2000)

5. Y. A. Horr, M. Arif, A. Kaushik, A. Mazroei, M. Katafygiotou, E. Elsarrag, Build. Environ. 105 (2016)

6. P. M. Bluyssen, The Indoor Environment Handbook: How to make buildings healthy and comfortable (London, UK, Earthscan, 2009)

7. A. Szczurek. M. Maciejewska, M. Teuerle, A. Wyłomańska, Physica A, 420 (2015)

8. P. M. Bluyssen, Build. Environ. 45 (2010)

9. A. Al-Hemoud, L. Al-Awadi, A. Al-Khayat, W. Behbehani, Build. Environ. 137, (2018)

10. A. Szczurek, A. Dolega, M. Maciejewska, Energ. Buildings, 158 (2018)

11. A. Szczurek, M. Maciejewska, T. Pietrucha, Energ. Buildings, 147 (2017)

12. S. H. Ryu, H. J. Mon, Build. Environ. 107 (2016)

13. T. H. Pedersen, K. U. Nielsen, S. Petersen, Build. Environ. 115 (2017) 